\title{
Correction to: Methodologies and Intelligent Systems for Technology Enhanced Learning, 9th International Conference, Workshops
}

\author{
Elvira Popescu, Ana Belén Gil, Loreto Lancia, \\ Luigia Simona Sica, and Anna Mavroudi
}

\section{Correction to: \\ E. Popescu et al. (Eds.): Methodologies and Intelligent Systems \\ for Technology Enhanced Learning, 9th International \\ Conference, Workshops, AISC 1008, https://doi.org/10.1007/978-3-030-23884-1}

In the original version of the book, the following belated corrections in the chapters have been incorporated:

1. In chapter 8, the author names Berardi Anna, Galeoto Giovanni, Tofani Marco, Mangone Massimiliano, Ratti Serena, Danti Arianna, Sansoni Julita, Marquez Maria Auxiliadora have been changed to Anna Berardi, Giovanni Galeoto, Marco Tofani, Massimiliano Mangone, Serena Ratti, Arianna Danti, Julita Sansoni and Maria Auxiliadora Marquez.

2. In chapter 9, the author names Salviani Silvia, Tofani Marco, Fabbrini Giovanni, Leo Antonio, Berardi Anna, Sansoni Julita, Galeoto Giovanni have been changed to Silvia Salviani, Marco Tofani, Giovanni Fabbrini, Antonio Leo, Anna Berardi, Julita Sansoni and Giovanni Galeoto.

The corrected chapter has been updated with the changes.

The updated version of these chapters can be found at https://doi.org/10.1007/978-3-030-23884-1_8 https://doi.org/10.1007/978-3-030-23884-1_9 